Original Research Paper

\title{
Sodium L-Glutamate-Induced Physiological Changes in Lactobacillus Brevis NCL912 During GABA Production Under Acidic Conditions
}

\author{
Gui-Dong Huang, Jian Mao, Zhongwei Ji and Askaer Alati \\ State Key Laboratory of Food Science and Technology, School of Food Science and Technology, Jiangnan \\ University, Wuxi, PR China
}

\author{
Article history \\ Received: 29-04-2014 \\ Revised: 12-09-2014 \\ Accepted: 01-12-2014 \\ Corresponding Author: \\ Jian Mao, \\ State Key Laboratory of Food \\ Science and Technology, \\ School of Food Science and \\ Technology, Jiangnan \\ University, Wuxi 214122, \\ China \\ Tel: +86-510-8532-9062, Fax: \\ $+86-510-8591-2155$, \\ E-mail: maojiand417@163.com
}

\begin{abstract}
Previous studies showed that $\gamma$-aminobutyric acid (GABA) can protect some GABA-producing bacteria against acid stress through glutamate decarboxylation. However, the acid stress mechanism is a comprehensive network related to numerous genes and proteins. Other than the Glutamate Decarboxylase (GAD)/GABA antiporter system, limited information is available about the physiological modifications that occur in Lactobacillus brevis during GABA production under acidic conditions. Therefore, this study aims to investigate the physiological modifications that occur in a GABA-producing L. brevis NCL912 during GABA production under acidic conditions. The differential protein expression of $L$. brevis NCL912 under different culture conditions was determined by using proteome analysis. Eleven of the protein spots were identified by mass spectrometry. Among the 11 proteins, 10 were upregulated, whereas one was downregulated. The function of downregulated protein was unknown. The upregulated proteins were involved in stress response, protein synthesis, quorum sensing, glycolysis and membrane lipid synthesis. Stress response, protein synthesis, glycolysis-related proteins are general acid stress proteins, while LuxS-dependent quorum sensing system and membrane lipid synthesis-related proteins might be induced by sodium L-glutamate during GABA production under acid stress. In conclusion, sodium L-glutamate might trigger other acid-tolerance responses, except the GAD/GABA system in L. brevis NCL912 during GABA production under acidic conditions.
\end{abstract}

Keywords: Lactobacillus brevis NCL912, Sodium L-Glutamate, $\gamma$ Aminobutyric Acid, Differentially Expressed Proteins, Acid Stress

\section{Introduction}

Lactobacillus is generally regarded as safe to humans. These bacteria serve an important function in the food industry. Researchers have exerted considerable effort to select more new Lactobacillus strains with functional properties for fermented products because of the positive effects of lactobacillus on human health. However, only a limited number of Lactobacillus can be used in commercial food products or probiotics because Lactobacillus performance under optimal laboratory conditions might be difficult to achieve under typical processing conditions. For instance, starter strains have to resist adverse conditions encountered in industrial processes (i.e., low and high temperatures, osmotic stress, etc.) (Zamfir and Grosu-Tudor, 2014). As probiotics, Lactobacillus must pass the natural low $\mathrm{pH}$ barrier in the stomach to ensure that an adequate number will reach the target site to induce a beneficial effect. Therefore, acid tolerance is one of the main characteristics that Lactobacillus should possess under stress conditions. Mechanisms contributing to the capacity of Lactobacillus to tolerate acidic $\mathrm{pH}$ are essential to the production and functionality of a probiotic culture (Azcarate-Peril et al., 2004).

The $\gamma$-aminobutyric acid (GABA) is a non-protein amino acid that is widely distributed in nature and can be found in microorganisms, plants and animals. GABA is also the chief inhibitory neurotransmitter in the central nervous system of mammals (Li et al., 2008). Studies showed that GABA is important to some bacteria, (such as E.coli, Listeria monocytogenes) that produce GABA through glutamate decarboxylation under acid stress (Tramonti et al., 2006; Feehily et al., 2014). Glutamate decarboxylase (GAD) (EC 4.1.1.15) is a pyridoxal 5'- 
phosphate-dependent enzyme that catalyzes the protonconsuming conversion of L-glutamate or glutamine to GABA (Tramonti et al., 2006). When bacteria are exposed to environments that cause acid stress, cytosolic acidification stimulates GAD activity and protons $(\mathrm{H}+)$ are consumed in the cytoplasm, thus increasing internal $\mathrm{pH}$. The product of alkalescent GABA is exported from the cell via the antiporter system. This product increases external $\mathrm{pH}$ and helps maintain a neutral cytoplasmic $\mathrm{pH}$ when external $\mathrm{pH}$ decreases. The GAD/GABA antiporter system is regarded as a defense mechanism of bacteria against acidic environments (Richard and Foster, 2004; Siragusa et al., 2007; Small and Waterman, 1998).

Some studies reported that Lactobacillus paracasei, Lactobacillus brevis, Lactobacillus reuti and Lactobacillus buchneri are also assayed in culture media and/or in food matrices for GABA synthesis (Gobbetti et al., 2010; Su et al., 2011). In our previous study, Lactobacillus brevis NCL912 significantly converted sodium L-glutamate (L-MSG) to GABA and GAD activity was significantly enhanced under acid stress $(p<0.05)$. The putative acid stress response mechanism is referred to as the GAD/GABA antiporter system (Huang et al., 2012). However, the acid stress mechanism is a comprehensive network related to numerous genes and proteins. Except for the GAD/GABA antiporter system, little is known about the physiological modifications that occur in Lactobacillus brevis during GABA production under acid stress. Therefore, this study investigates the physiological modifications that occur in GABA-producing L. brevis NCL912 during GABA production under acidic conditions. The results may provide new insights into the inducible acid stress response mechanisms of this bacterium.

\section{Materials and Methods}

\section{Strain, Media and Culture Conditions}

High GABA-producing L. brevis NCL912 (CCTCCM 208054) was isolated from paocai, a Chinese traditional fermented vegetable, in our laboratory. Modified MRS broth (MMRS) was used as the culture medium, which contained in $\mathrm{g} \mathrm{L}^{-1}$, glucose, 50; yeast extract, 12.5 ; soya peptone, $12.5 ; \mathrm{MgSO}_{4} \cdot 7 \mathrm{H}_{2} \mathrm{O}, 0.2$; $\mathrm{MnSO}_{4} \cdot 4 \mathrm{H}_{2} \mathrm{O}, 0.05$ and Tween $80,1 \mathrm{~mL} \mathrm{~L}^{-1}$. The initial L-MSG concentration in the medium was $0.1 \mathrm{~mol} \mathrm{~L}^{-1}$ (Huang et al., 2012; Li et al., 2008). The $\mathrm{pH}$ of the medium was adjusted with hydrochloric acid to $\mathrm{pH} 4.0$.

\section{Measurement of Growth Curves, GABA and $p H$}

Strains were propagated overnight twice in MMRS at $32^{\circ} \mathrm{C}$. The strains $\left(4.3 \times 10^{8}\right)$ were then added into the MMRS with or without L-MSG at pH $4.0( \pm 0.1)$. Samples were obtained every $4 \mathrm{~h}$. After that, cell growth, the variations in $\mathrm{pH}$ and GABA yield were measured. GABA yield was determined by pre-staining paper chromatography (Li et al., 2009).
Protein Extraction and Two-Dimensional Gel Electrophoresis (2-DE)

The strain was incubated in the medium with or without L-MSG at $\mathrm{pH} 4.0$ for $4 \mathrm{~h}$. The cells were then collected by centrifugation at $8000 \times \mathrm{g}$ for $10 \mathrm{~min}$, washed twice with $\mathrm{pH} 7.0$ phosphate-buffered saline and then dissolved in lysis buffer containing $7 \mathrm{M}$ urea, $2 \mathrm{M}$ thiourea, $\quad 4 \% \quad 3-[$ (3-cholamidopropyl) dimethylammonio)]-1-propanesulfonate, 1\% Isoelectric Focusing (IEF) buffer and $65 \mathrm{~m}$ Mdithiothreitol (DTT). The lysates were centrifuged at $12,000 \times \mathrm{g}$ for $60 \mathrm{~min}$ at $4^{\circ} \mathrm{C}$ and the supernatants were stored at $-80^{\circ} \mathrm{C}$ until further use. Protein concentration was estimated using the Bradford assay.

A $200 \mu \mathrm{g}$ protein sample was subjected to firstdimension IEF and loaded on $\mathrm{pH} 3.0 \sim 10.0$ at $18 \mathrm{~cm}$ nonlinear Immobilized pH Gradient (IPG) strip (SigmaAldrich, St Louis, USA). IEF was carried out using the EttanIPGphor II IEF system (GE Healthcare, Piscataway, USA) at $20^{\circ} \mathrm{C}$ as follows: $6 \mathrm{~h}$ at $40 \mathrm{~V}$ (active rehydration), $6 \mathrm{~h}$ at $100 \mathrm{~V}, 0.5 \mathrm{~h}$ at $500 \mathrm{~V}, 1 \mathrm{~h}$ at $1,000 \mathrm{~V}, 1 \mathrm{~h}$ at $2,000 \mathrm{~V}$, $1.5 \mathrm{~h}$ at $10,000 \mathrm{~V}$ and $60,000 \mathrm{Vh}$ at $10,000 \mathrm{~V}$. The minimal Vh applied was at least 60,000 units. After IEF, the strips were equilibrated for $15 \mathrm{~min}$ in an equilibration buffer (0.05 M Tris- $\mathrm{HCl} \mathrm{pH} 8.8,6 \mathrm{M}$ urea, 20\% glycerol, 2\% SDS) supplemented with $2 \%$ DTT for disulfide bridge reduction. The strips were supplemented with $2.5 \%$ iodoacetamide for an additional $15 \mathrm{~min}$ in the same equilibration buffer for cysteine alkylation. A 2D SDSPAGE was run in $12 \%$ polyacrylamide gels using the Bio-Rad Protean II xi cell (Bio-Rad, California, USA). Triplicate electrophoreses were performed to ensure reproducibility for each protein sample.

Gels were stained with silver, scanned using Image Scanner II (GE healthcare, Piscataway, USA) and then analyzed with Imagemaster 2D Elite 5.0 software (GE healthcare, Piscataway, USA). The chemicals for 2D electrophoresis were purchased from Sigma-Aldrich ( $\mathrm{St}$ Louis, USA). The significant and differentially expressed protein spots $(p<0.05)$ with two fold or more increased or decreased intensity in the medium with or without L-MSG were selected and subjected to further identification using an Applied BiosystemSciex 4800 Matrix-Assisted Laser Desorption/Ionization (MALDI) Time-of-Flight (TOF)/TOF Mass Spectrometer (MS) (Applied Biosystems, Carlsbad, USA).

\section{Protein Digestion}

Silver-stained protein spots were excised from the gel, washed with MilliQ water for $10 \mathrm{~min}$ and then destained in a 1:1 solution of $100 \mathrm{mM}$ ammonium bicarbonate and acetonitrile. After hydrating with acetonitrile and drying in a SpeedVac, all of the gel pieces were incubated with $20 \mu \mathrm{g}$ $\mathrm{mL}^{-1}$ sequencing-grade porcine trypsin (Promega, Madison USA) in $25 \mathrm{mM} \mathrm{NH} \mathrm{NCO}_{3}$ overnight at $37^{\circ} \mathrm{C}$. The supernatants were transferred into a $200 \mu \mathrm{L}$ microcentrifuge tube and the gels were extracted once with extraction buffer 
(67\% acetonitrile containing 1\% trifluoroacetic acid). The peptide extract and the supernatant were pooled and completely dried by Speed Vac.

\section{Mass Spectrometry Analysis and Database Searching}

Protein digestion extracts (tryptic peptides) were resuspended with $5 \mu \mathrm{L} 0.1 \%$ trifluoroacetic acid. The peptide samples were mixed (1:1 ratio) with a saturated solution of alpha-cyano-4-hydroxycinnamic acid in 50\% acetonitrile/water containing $0.1 \%$ trifluoroacetic acid; $0.8 \mu \mathrm{L}$ liquid was spotted onto the stainless steel sample target plates. Mass spectrometry analysis was conducted using MALDI TOF/TOF MS. The instrument was calibrated using a CalMix5 standard in reflection positive-ion mode (ABI4700 Calibration Mixture). The MS and MS/MS data were interpreted and processed using the Global Proteome Server Explorer ${ }^{\mathrm{TM}}$ software (Version 3.6, Applied Biosystems, Carlsbad, USA). The MS spectra were obtained from each sample spot by accumulating 600 to 800 laser shots in an 800 to 4000 mass range. On the other hand, the MS/MS spectra, which were the five most abundant precursor ions per sample, were selected for subsequent fragmentation. About 900 to 1200 laser shots were accumulated per precursor ion. The 20 most intense precursors per spot were selected with a minimum signal-to-noise ratio of 50 .

A combined $\mathrm{MS}$ and $\mathrm{MS} / \mathrm{MS}$ search was first performed against the NCBI database without taxonomic restriction using the MASCOT search engine (Version 2.1, Matrix Science, London, UK). Database search parameters were set as follows: trypsin of the digestion enzyme, one missed cleavage, $p<0.05$ significance threshold, partial modification of cysteine carboamidomethylated and methionine oxidized, nonefixed modifications, $50 \mathrm{ppm}$ peptide mass tolerance and MS/MS tolerance of 0.25 Da. Known contaminant ions (keratin) were excluded. MASCOT protein scores (based on combined MS and MS/MS spectra) greater than 51 were considered to be statistically significant $(p<0.05)$. The individual MS/MS spectrum with a statistically significant (confidence interval $>95 \%$ ) best ion score (based on MS/MS spectra) was also accepted.

\section{Verification at the Transcription Level by Real-Time Fluorescence Quantitative PCR}

Differentially expressed proteins required further validation at the mRNA level. The mRNA expression levels of selected proteins were determined using RealTime Fluorescence Quantitative Polymerase Chain Reaction (RTFQ-PCR). The total RNA was extracted using a TRIzol reagent (Tiangen Biotech, Beijing, China). The Super Script III first-strand synthesis kit was used for reverse transcription-PCR (Invitrogen, Carlsbad, USA). The gene-specific primer pairs used for RTFQ-PCR were designed as shown in Table 1. The RTFQ-PCR was performed using the FTC-2000 Realtime PCR System (Funglyn, Shanghai, China) and was analyzed with a Fast Start Universal SYBR Green
Master (Roche, Basel, Switzerland) according to the manufacturer's instructions. Relative gene expression levels were determined with the comparative threshold cycle $\left(\Delta \Delta \mathrm{C}_{\mathrm{t}}\right)$ method and $16 \mathrm{~S}$ rRNA gene was used as the endogenous reference gene.

\section{Statistical Analysis}

All of the experiments were done in triplicate. Data are expressed as mean \pm S.D. $(n \geq 3)$. Statistical comparisons were performed with SPSS 17.0. One-way ANOVA and student $t$-test were carried out to determine statistical significance, wherein $p<0.05$ was considered statistically significant.

\section{Results}

Growth Curves of L. brevis NCL912, GABA Yield and $\mathrm{pH}$ Change in the Medium with or Without LMSG at $\mathrm{pH} 4.0$

L. brevis NCL912 was incubated in the medium with or without L-MSG at $\mathrm{pH} 4.0$ for $36 \mathrm{~h}$. The growth curves, production of GABA and $\mathrm{pH}$ change were measured and results are shown in Fig. 1. L. brevis NCL912 had similar growth patterns in the medium with or without L-MSG. The exponential phase of $L$. brevis NCL912 was from $4 \mathrm{~h}$ to 24 $\mathrm{h}$ and the $\mathrm{OD}_{600 \mathrm{~nm}}$ of the strain in the medium with L-MSG was higher than that in the medium without L-MSG at the same growth period. This result suggested that L-MSG might improve the growth of $L$. brevis NCL912. In the medium with L-MSG, GABA yields increased from 0.72 to $10.46 \mathrm{~g} \mathrm{~L}^{-1}$ and $\mathrm{pH}$ was $3.99,4.21,4.51,5.18$ and 6.42 at different culture times $(0,4,8,12$ and $24 \mathrm{~h})$, respectively (Fig. 1.). In the medium without L-MSG, GABA content slightly increased (from 0.68 to $1.14 \mathrm{~g} \mathrm{~L}^{-1}$ ) before $8 \mathrm{~h}$, which might be related to the minute amount of glutamate in the medium. Thus, $\mathrm{pH}$ slightly increased at $4 \mathrm{~h}$, with $3.96,4.10,3.91,3.73$ and 3.41 at different culture times $(0$, 4, 8, 12 and 24 h), respectively. These results (Fig. 1.) suggested that $L$. brevis NCL912 tolerated acid condition by GABA accumulation and increasing the $\mathrm{pH}$ of the medium. However, at $4 \mathrm{~h}$, similar $\mathrm{pH}$ and GABA content were observed and the $\mathrm{OD}_{600 \mathrm{~nm}}$ in the medium with L-MSG was higher than that in the medium without L-MSG. This result suggested that GABA accumulation might not be the only response of L. brevis NCL912 in resisting acid stress.

\section{Differentially Expressed Proteins of L. Brevis NCL912 during GABA Production under Acidic Conditions}

Differentially expressed proteins of $L$. brevis NCL912 during GABA production under acidic conditions were determined in the study. The total proteins of high GABA-producing L. brevis NCL912 in the medium with or without L-MSG at $\mathrm{pH} 4.0$ were separated by 2 -DE. Image analysis revealed 26 protein spots, which clearly showed differential expressions under the two culture conditions (Fig. 2). 


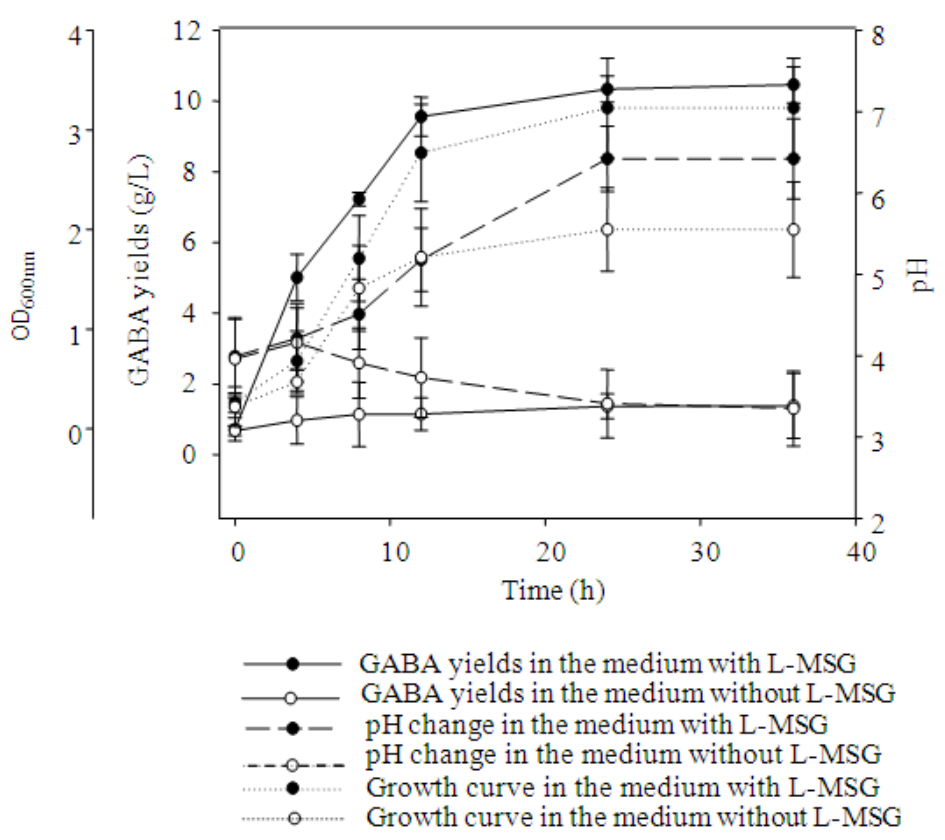

Fig. 1. The growth curves of L. brevis NCL912, GABA yield and $\mathrm{pH}$ change in the medium with or without L-MSG at pH 4.0 (Huang et al., 2012)

Table 1. Primer sequences used for RTFQ-PCR

\begin{tabular}{|c|c|c|c|c|}
\hline Gene & Accession no. & Primers direction & Reverse primer sequence ( $5^{\prime}$ to $\left.3^{\prime}\right)$ & Amplicon size (bp) \\
\hline \multirow[t]{2}{*}{ 16s rRNA } & 4413327 & Sense & CC TAC GGG AG G CAG CAG & \\
\hline & & Antisense & ATT ACC GCG GCT GCT GG & 103 \\
\hline \multirow[t]{2}{*}{ UspA } & 4414418 & Sense & GTGGTGCCGTTAGTGGTGAC & \\
\hline & & Antisense & CGGGCAATCACGGGTTTA & 148 \\
\hline \multirow[t]{2}{*}{ GrpE } & 4414470 & Sense & TCAGCAACTGGCTAAGGACG & \\
\hline & & Antisense & GGTTACGCTTCAGGGCATCT & 146 \\
\hline \multirow[t]{2}{*}{ S-rib } & 4413194 & Sense & AGAAACTGGGACGAAGGGTG & \\
\hline & & Antisense & CGCTAATAAATGCTCGATGGTA & 112 \\
\hline \multirow[t]{2}{*}{ ndse } & 4414358 & Sense & AGCAACTCGCAGCAACTGG & \\
\hline & & Antisense & ATCTTCAGCGGTCCCTAACAG & 122 \\
\hline
\end{tabular}

Eleven of the 26 spots were identified by matrix-assisted laser desorption/ionization time-of-flight/time-of-flight mass spectrometry. The expression levels of spots $1,4,5$, $6,7,9,10,15,20,22$ were upregulated in the medium with L-MSG, whereas that of spot 21 was downregulated in the medium without L-MSG (Table 2).

\section{Cellular Localization Analysis of Identified Proteins \\ PSORTb \\ version}

(http://www.psort.org/psortb/index.html) was used to predict the cellular localization of the 11 identified proteins (Table 2). Six proteins localized in the cytoplasm accounting for $54.5 \%$ of the total proteins. RNA-Binding Protein (RBP) was in the outermembrane, hypothetical protein was in the periplasm and other three proteins had an unknown cellular location (Table 3 ).

\section{Classification and Function Analysis of Identified Proteins}

To determine the acid stress response mechanisms of that occurs in L. brevis NCL912 during GABA production under acidic conditions, biological process and molecular functional data of the identified proteins were obtained from the UniProtKB protein knowledgebase (http://www.uniprot.org). The identified upregulated proteins were related to stress response, protein synthesis, glycolysis, cell membrane formation and cell communication. The function of the hypothetical protein BRAFLDRAFT_64486 (21) was unclear (Table 3 ).

\section{RTFQ-PCR Analysis of Selected Proteins}

The expression levels of the UspA family nucleotide-binding protein (UspA), molecular chaperone GrpE, S-ribosylhomocysteinase (LuxS) and Nucleoside-Diphosphate-Sugar Epimerase (NDSE) were estimated at the mRNA level by RTFQ-PCR. The RTFQ- PCR results indicated that the RTFQ-PCR analysis data matched the protein expression levels from 2-DE (Fig. 3.). The mRNA expression levels of UspA protein, GrpE and NDSE significantly increased in the medium with L-MSG $(p<0.05)$. 


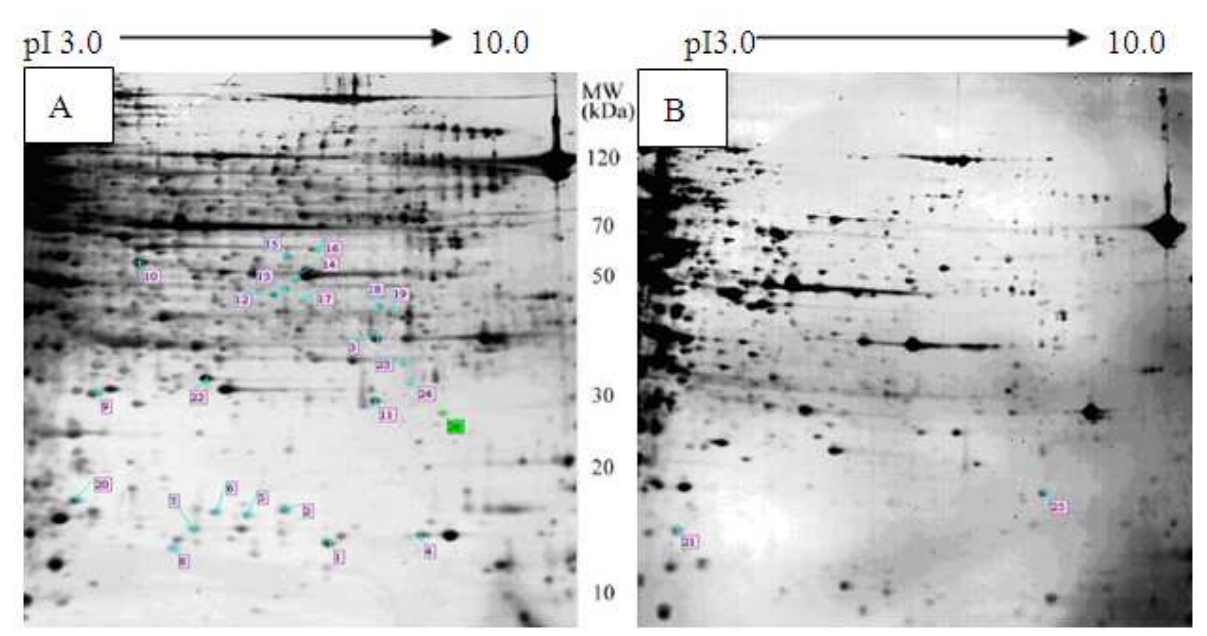

Fig. 2. 2-DE maps of proteins extracted from L. brevis NCL912 in the medium (A) with or (B) without L-MSG at pH 4.0. The spots were visualized bysilverstaining. Differentially expressed protein spots for each treatment were marked with numbers (Huang et al., 2012)

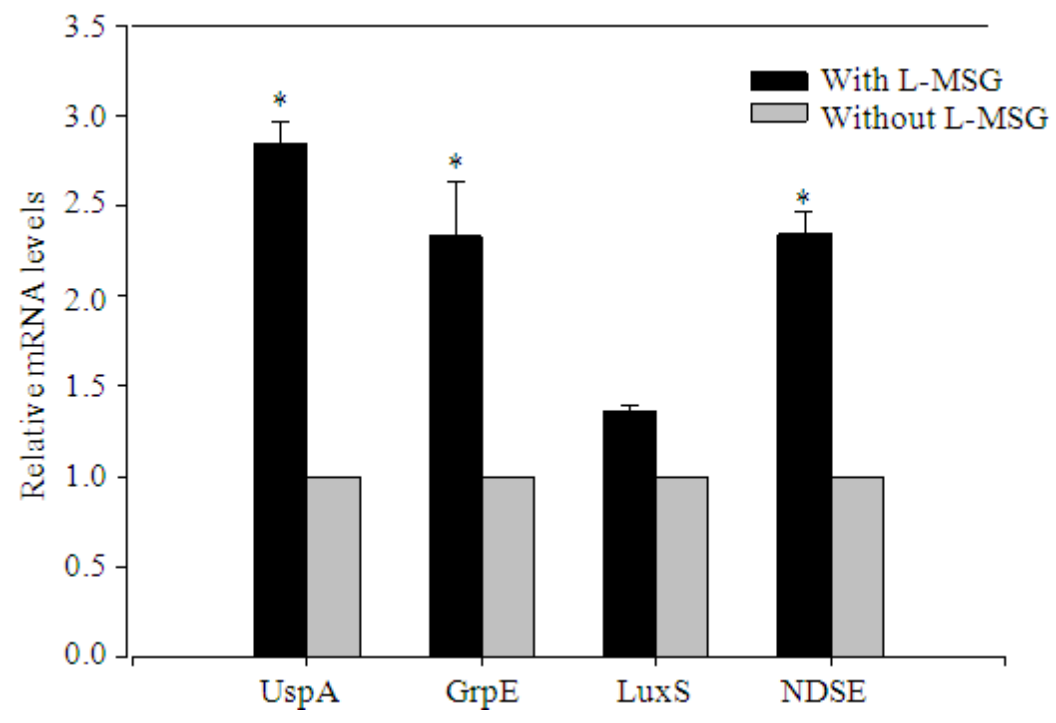

Fig. 3. Relative mRNA levels of selected differentially expressed proteins in L. brevis NCL912 as revealed by RTFQ-PCR Significant differences in gene expression are indicated with asterisks $(*)(p<0.05)$ UspA, UspA family nucleotide-binding protein; GrpE, molecular chaperone GrpE; LuxS, S-ribosylhomocysteinase; NDSE, nucleoside-diphosphate-sugar epimerase

Table 2. Identified differentially expressed proteins of L. brevis NCL912 during GABA production under acid conditions

\begin{tabular}{|c|c|c|c|c|c|c|c|c|c|}
\hline Spots & Protein & Accession no. & Locus & $\begin{array}{l}\text { Theor. } \\
\mathrm{Mr}(\mathrm{Da}) / \mathrm{Pi}\end{array}$ & $\begin{array}{l}\text { Number of } \\
\text { peptides matched }\end{array}$ & $\begin{array}{l}\text { Protein } \\
\text { score }\end{array}$ & $\begin{array}{l}\text { Sequence } \\
\text { coverage (\%) }\end{array}$ & $\begin{array}{l}\text { Total ion } \\
\text { score }\end{array}$ & $\begin{array}{l}\text { Cellular } \\
\text { localization }\end{array}$ \\
\hline 1 & UspA & gi|116332842 & YP_794369.1 & $16474.8 / 6.82$ & 22 & 234 & 14 & 109 & Unknown \\
\hline 4 & UspA & gi||116334159 & YP_795686 & $16883 / 9.16$ & 16 & 86 & 10 & 60 & $\begin{array}{l}\text { Cytoplasm } \\
\text { Outer }\end{array}$ \\
\hline 5 & RBP & gi|221056514 & XP_002259395.1 & $183415.4 / 5.79$ & 12 & 92 & 6 & 55 & Membrane \\
\hline 6 & PBP & gi|116332861 & YP_794388.1 & $18565.4 / 5.71$ & 16 & 72 & 9 & 58 & Unknown \\
\hline 7 & LuxS & gi|116333010 & YP_794537.1 & $17653.8 / 5.48$ & 18 & 80 & 9 & 61 & Cytoplasm \\
\hline 9 & GrpE & gi|1 16333936 & YP_795463.1 & 21231.7/4.6 & 11 & 101 & 5 & 52 & Cytoplasm \\
\hline 10 & GAPDH & gi|42784288 & NP_981535.1 & $35757.3 / 5.37$ & 45 & 320 & 13 & 209 & Cytoplasm \\
\hline 15 & GPAT & gi|116333592 & YP_795119.1 & $36704 / 5.48$ & 22 & 88 & 6 & 88 & Cytoplasm \\
\hline 20 & EF-Tu & gi|116333991 & YP_795518 & $43612.1 / 4.73$ & 16 & 219 & 4 & 154 & Cytoplasm \\
\hline 21 & HPB & gi|219408088 & XP_002201859.1 & $729515.9 / 5.62$ & 14 & 94 & 6 & 63 & Periplasm \\
\hline 22 & NDSE & gi|116332989 & YP_794516.1 & $22722.7 / 5.47$ & 14 & 119 & 6 & 100 & Unknown \\
\hline
\end{tabular}

UspA, UspA family nucleotide-binding protein; GrpE, molecular chaperone GrpE; LuxS, S-ribosylhomocysteinase, NDSE, nucleoside-diphosphatesugar epimerase; RBPs, RNA-binding proteins; EF-Tu, elongation factor Tu; GAPDH, glyceraldehyde-3-phosphate dehydrogenase; GPAT, putative glycerol-3-phosphate acyltransferasePlsX, PBP, phospholipid-binding protein; HPB, Hypothetical protein BRAFLDRAFT_64486 
Table 3.Cellular localization and function of identified differentially expressed proteins of L. brevis NCL912 during GABA production under acid conditions

\begin{tabular}{|c|c|c|c|c|}
\hline Protein & Spots & $\begin{array}{l}\text { Cellular } \\
\text { localization }\end{array}$ & Biological process & Molecular Function annotation \\
\hline \multicolumn{5}{|l|}{ pH 4.0 stress-induced proteins } \\
\hline UspA & 1 & Unknown & Response to stress & $\begin{array}{l}\text { Required for resistance to DNA- } \\
\text { damaging agents; Involved in stress } \\
\text { response }\end{array}$ \\
\hline UspA & 4 & Cytoplasm & Response to stress & $\begin{array}{l}\text { Required for resistance to DNA- } \\
\text { damaging agents; Involved in stress } \\
\text { response }\end{array}$ \\
\hline GrpE & 9 & Cytoplasm & Response to stress; protein folding & Chaperone \\
\hline \multicolumn{5}{|l|}{ Protein synthesis-related proteins } \\
\hline RBP & 5 & OuterMembrane & Translation & Nucleic acid binding \\
\hline EF-Tu & 20 & Cytoplasm & Protein biosynthesis & Translation elongation factor activity \\
\hline \multicolumn{5}{|l|}{ Glycolysis-related proteins } \\
\hline GAPDH & 10 & Cytoplasm & Glucose metabolic process & Oxidoreductase activity \\
\hline \multicolumn{5}{|l|}{ Quorum sensing-related proteins } \\
\hline LuxS & 7 & Cytoplasm & Quorum sensing & S-ribosylhomocysteine lyase activity \\
\hline \multicolumn{5}{|l|}{ Membrane lipid synthesis-related proteins } \\
\hline GPAT & 15 & Cytoplasm & Fatty acid biosynthetic process & $\begin{array}{l}\text { Transferase activity, transferring acyl } \\
\text { groups other than amino-acyl groups }\end{array}$ \\
\hline NDSE & 22 & Unknown & - & - \\
\hline $\begin{array}{l}\text { PBP } \\
\text { pH } 5.0 \text { Unknown function }\end{array}$ & 6 & Unknown & - & - \\
\hline HPB & 21 & Periplasm & - & - \\
\hline
\end{tabular}

The LuxS mRNA expression level was also upregulated, although not significantly, in the medium with L-MSG (Fig. 3). These findings are attributed to the differences in the regulation mechanisms, such as synthesis and degradation rates, that act on mRNA and protein synthesis and ultimately affect the molecular levels (Jianke et al., 2010).

\section{Discussion}

In this present study, L. brevis NCL912 could grow at $\mathrm{pH}$ 4.0, GABA yields and $\mathrm{pH}$ increased with culture time. This finding suggested that the acid tolerance properties of L. brevis NCL912 might be related to $\mathrm{pH}$ increase and GABA accumulation. The putative acid stress response mechanism is referred to as the GAD/GABA antiporter system and the mechanism was reported in our previous study (Huang et al., 2012). However, the strain grew better in the medium with LMSG than in the medium without L-MSG at $4 \mathrm{~h}$, during which the $\mathrm{pH}$ and GABA content were similar. This result suggested that the GAD/GABA antiporter system might not be the only response of L. brevis NCL912 in resisting acid stress. At present, except for the GAD/GABA antiporter system, little is known about the physiological modifications that occur in L. brevis NCL912during GABA production under acid conditions. Therefore, the physiological modifications that occur in GABA-producing L. brevis NCL912 during GABA production under acidic conditions were investigated in this study. Here, the expressions of stress response, protein synthesis, glycolysis, LuxS-dependent QS system and membrane lipid synthesis-related proteins were up-regulated. All these suggested that stress response, protein synthesis, glycolysis-related proteins are general acid stress proteins, while LuxS-dependent QS system and membrane lipid synthesis-related proteins might be induced by L-MSG during GABA production under acid stress. The up-regulation of these proteins protects $L$. brevis NCL912 against acid stress. However, proteomic analyses failed to detect GAD among the differentially expressed proteins, although this result does not absolutely exclude that GAD expression was modulated by glutamate because GAD might be among the unidentifiable spots in very low amounts or because the differential expression level of GAD is less than twofold under L-MSG-stimulated conditions than under control conditions. The result is consistent with the mRNA levels of gadA in L. brevis NCL912 reported by Li et al. (2013), who found that gadA is only GAD encoding gene in L. brevis NCL912. The mRNA levels of gadA in L. brevis NCL912 were almost identical during the $0 \mathrm{~h}$ to $12 \mathrm{~h}$ culture period in the medium with or without glutamate.

\section{Stress Response Proteins}

Proteomic results indicated that the expressions of UspA protein and GrpE upregulated in this study. Previous studies showed that UspA protein and GrpE are essential stress-response proteins and are both essential components of the acid stress response network (Chen et al., 2006; Licandro-Seraut et al., 2008; Zhang and Griffiths, 2003; Zininga and Shonhai, 2014). 
UspA serves a regulatory function in biofilm formation and DNA repair, as well as affects the motility and adhesion of bacterium under stress conditions (Chen et al., 2006). Meanwhile, GrpE contributes to the maturation of newly synthesized polypeptides, promotion of protein refolding and degradation of denatured proteins under stress (Fukuda et al., 2002). The observed over expression of UspA and GrpE suggests that L. brevis NCL912 tolerates acid stress conditions during GABA production under acidic conditions by regulating the expression of L. brevis NCL912 stress-response proteins.

\section{Protein Synthesis-Related Proteins}

RNA-Binding Proteins (RBPs) and elongation factor $\mathrm{Tu}(\mathrm{EF}-\mathrm{Tu})$ in L. brevis NCL912, which are related to protein synthesis, were upregulated in this study. RBPs serve key functions in the post-transcriptional regulation of gene expression that involves editing, degradation and translation (Lee and Schedl, 2006). These processes can occur at many different steps during RNA metabolism. EF-Tu, one of the prokaryotic elongation factors that participate in the protein translation of ribosome, is remarkably conserved and is responsible for achieving accurate ribosome translation. This factor transiently mediates the selection and binding of the cognate aminoacyl-tRNA to the acceptor site of the ribosome, thus serving a crucial function in protein synthesis (Dallo et al., 2012). The upregulation of these proteins indicates protein synthesis is activated. Such activation may have protected L. brevis NCL912 by allowing it to tolerate acidic conditions.

\section{Glycolysis-Related Proteins}

The expression of glyceraldehyde-3-phosphate dehydrogenase (GAPDH), a key enzyme in glycolysis that reversibly catalyzes the oxidation and phosphorylation of glyceraldehyde-3-phosphate to 1,3diphospho-glycerate, was altered under different conditions in the study. This finding indicates that glycolysis may be an essential metabolic pathway for anaerobic bacteria and facultative anaerobes in response to various stressful conditions. GAPDH is also a multifunctional protein that affects membrane fusion, gene transcription, DNA replication, DNA repair and nuclear RNA export (Wouters et al., 2000).

The observed upregulation of GAPDH suggests that L. brevis NCL912 obtained additional energy for growth and survival by affecting the glycolysis pathway during GABA production under acidic conditions. The result is the same as that observed in Lactococuss lactic NCDO 2118 (Mazzoli et al., 2010).

\section{Quorum Sensing-Related Proteins}

Quorum Sensing (QS) is a system of stimulus and response correlated to population density. It is the best characterized as a means of communication within bacteria (Sun et al., 2014). Many species of bacteria use
QS to coordinate gene expression and regulate quorum behavior according to the density of their local population which allows bacteria to rapidly adapt to surrounding changes (Gölz et al., 2012).

Bacteria use QS constitutively produce and secrete certain signaling molecules: (1) production of small biochemical signal molecules by bacterial cell; (2) release of the signal molecules into the surrounding environment; and (3) recognition of the signal molecules by specific receptors once they exceed a threshold concentration, leading to (4) changes in gene regulation (Galloway et al., 2011). In the Gram-negative bacterium, autoinducer-2 (AI-2)-mediated system is the main signaling system of QS. The enzyme LuxS, which is widely conserved throughout the bacterial kingdom, is responsible for AI-2 biosynthesis (Plummer, 2012). LuxS catalyzes the cleavage of the thioether bond in $S$ ribosylhomocysteine (SRH) to produce homocysteine and 4,5-dihydroxy-2,3-pentanedione (DPD), which undergoes spontaneous rearrangements to form a variety of DPD derivatives that interconvert and exist in equilibrium (known as the AI-2 pool). AI-2 responses in bacteria can be triggered by different members of the AI2 compound pool (Rajan et al., 2005).

In the present study, LuxS in L. brevis NCL912 were up-regulated during GABA production under acidic conditions. Therefore, activated LuxS enzyme is assumed to produce more AI-2, the signal molecule is transported into the external environment to trigger QS system, which protects L. brevis NCL912 to tolerate low acid conditions. Moslehi-Jenabian et al. (2009) also considered that AI-2 signaling system is induced by acidic shock in probiotic strains of Lactobacillus spp.

\section{Membrane Lipid Synthesis-Related Proteins}

Cell membrane plays critical roles in the maintenance of cell structure and in the modulation of cell function which serves as a barrier of separating the interior of all cells from the outside environment. It is one of the most sensitive parts in response to stressful conditions. Stressful conditions could lead to the damage of cell membrane (Miajlovic and Smith, 2014). In this study, the expressions of Phospholipid-Binding Protein (PBP), glycerol-3-phosphate acyltransferase PlsX (GPAT), GAPDH and NDSE were up-regulated.

GAPDH and GPAT are the key enzymes of phospholipid synthesis. Phospholipid is the major component of cell membrane (Roy, 2009). GAPDH catalyzes dihydroxyacetone phosphate (DHAP) into glycerol-3-phosphate and DHAP is from the glycolytic pathway. GPAT transfers fatty acid from fatty acyl coenzyme A to glycerol-3-phosphate to produce lysophosphatidic acid (LysoPA). LysoPA is further acylated by a separate acyltransferase to produce Phosphatidic Acid (PA), a key intermediate in membrane 
phospholipid synthesis (Lu et al., 2006). PBP transfers phospholipid to cell membrane.

A previous report showed that NDSE catalyzes the interconversion of Uridine Diphosphate Galactose (UDP-Gal) and Uridine Diphosphate Glucose (UDPGlu). UDP-Gal serves a function in the biosynthesis of monogalactosyldiacyl glycerol (MGDG), a major galactolipid of cell membrane (Li et al., 2011).

In the present study, L-MSG induces the upregulations of PBP, GPAT, GAPDH and NDSE expressions in L. brevis NCL912 during GABA production under acidic conditions. The cluster of proteins is related to the synthesis of phospholipid and glycolipids which are the major components of cell membrane. That is, L-MSG may protect L. brevis NCL912 against acid stress by regulating lipid synthesis related to cell membrane formation.

\section{Conclusion}

In conclusion, the physiological changes observed in L. brevis NCL912 during GABA production under acidic conditions include the over expression of particular stress proteins, regulation of protein synthesis and cell communication and participation in glucose catabolism and cell membrane formation. These findings suggest that during GABA production under acidic conditions, L-MSG might trigger other acid stress response mechanisms aside from the GABA/GAD system.

\section{Acknowledgment}

Special thanks to Prof. Yu-sheng Cao for his helpful suggestions and assistance.

\section{Funding Information}

The present study was supported by the Selfdetermined Research Program of Jiangnan University (Project No.JUSRP11221) and Jiangsu Planned Projects for Postdoctoral Research Funds (No.1302105C).

\section{Author's Contributions}

The contribution made by each author to the research as follow:

Gui-Dong Huang: was responsible for the whole study and wrote paper.

Zhongwei Ji: Participated in qRT-PCR analysis.

Askaer Alati: Participated in the bio information analysis.

Jian Mao: Modified paper and was responsible for all the things.

\section{Conflict of Interest}

The authors declare no competing financial interest.

\section{Ethics}

All authors have read and approved the manuscript.

\section{References}

Azcarate-Peril, M., E. Altermann, R.L. Hoover-Fitzula, R. Cano and T. Klaenhammer, 2004. Identification and inactivation of genetic loci involved with Lactobacillus acidophilus acid tolerance. Applic. Environ. Microb., 70: 5315-5322. DOI: 10.1128/AEM.70.9.5315-5322.2004

Chen, W., K. Honma, A. Sharma and H.K. Kuramitsu, 2006. A universal stress protein of Porphyromonas gingivalis is involved in stress responses and biofilm formation. FEMS Microbiol. Lett., 264: 15-21. PMID: 17020544

Dallo, S.F., B. Zhang, J. Denno, S. Hong and A. Tsai et al., 2012. Association of acinetobacter baumannii EFTu with cell surface, outer membrane vesicles and fibronectin. Sci. World J., 2012: 128705-128705. PMID: 22666090

Feehily, C., A. Finnerty, P.G. Casey, C. Hill and C.G. Gahan et al., 2014. Divergent evolution of the activity and regulation of the glutamate decarboxylase systems in Listeria monocytogenes EGD-e and 10403S: Roles in virulence and acid tolerance. PLoS One, 9: e112649e112649. PMID: 25386947

Fukuda, D., M. Watanabe, Y. Aso, K. Sonomoto and A. Ishizaki, 2002. The groESL operon of the halophilic lactic acid bacterium Tetragenococcus halophila. Biosci Biotech Bioch., 66: 1176-1180.

PMID: 12092841

Galloway, W.R., J.T. Hodgkinson, S.D. Bowden, M. Welch and D.R. Spring, 2011. Quorum sensing in Gram-negative bacteria: Small-molecule modulation of AHL and AI-2 quorum sensing pathways. Chem. Rev., 111: 28-67. PMID: 21182299

Gobbetti, M., R.D. Cagno and M. De Angelis, 2010. Functional microorganisms for functional food quality. Crit. Rev. Food Sci. Nutr., 50: 716-727. PMID: 20830633

Gölz, G., S. Sharbati, S. Backert and T. Alter, 2012. Quorum sensing dependent phenotypes and their molecular mechanisms in Campylobacterales. Eur. J. Microbiol. Immunol., 2: 50-60. DOI: 10.1556/EuJMI.2.2012.1.8

Huang, G., C. Li and Y. Cao, 2012. Effect of sodium Lglutamate on growth and survival of Lactobacillus brevis NCL912 at different acidic pH. Ann. Microbiol., 62: 351-355. DOI: $10.1007 / \mathrm{s} 13213-011-0269-7$

Jianke, L., F. Mao, D. Begna, F. Yu and Z. Aijuan, 2010. Proteome comparison of hypopharyngeal gland development between Italian and royal jelly producing worker honeybees (Apis mellifera L.). J. Proteome. Res., 9: 6578-6594. PMID: 20882974 
Lee, M.H. and T. Schedl, 2006. RNA-binding proteins. Worm. Book, 18: 1-13.

DOI: 10.1895/wormbook.1.79.1

Li, C., Y. Wang, L. Liu, Y. Hu and F. Zhang et al., 2011. A rice plastidial nucleotide sugar epimerase is involved in galactolipid biosynthesis and improves photosynthetic efficiency. PLoS Genet., 7: e1002196-e1002196.

DOI: 10.1371/journal.pgen. 1002196

Li, H., D. Gao, Y. Cao and H. Xu, 2008. A high $\gamma$ aminobutyric acid-producing Lactobacillus brevis isolated from Chinese traditional paocai. Ann. Microbiol., 58: 649-653. DOI: $10.1007 / \mathrm{BF} 03175570$

Li, H., T. Qiu, Y. Cao, J. Yang and Z. Huang, 2009. Prestaining paper chromatography method for quantification of gamma-aminobutyric acid. J. Chromatogr. A, 1216: 5057-5060. PMID: 19446298

Li, H., W. Li, X. Liu and Y. Cao, 2013. gadA gene locus in Lactobacillus brevis NCL912 and its expression during fed-batch fermentation. FEMS Microbiol. Lett, 349: 108-116. PMID: 24164637

Licandro-Seraut, H., J. Gury, N.P. Tran, L. Barthelmebs and J.F. Cavin, 2008. Kinetics and intensity of the expression of genes involved in the stress response tightly induced by phenolic acids in Lactobacillus plantarum. J. Mol. Microb. Biotechn., 14: 41-47. PMID: 17957109

Lu, Y.J., Y.M. Zhang, K.D. Grimes, J. Qi and R.E. Lee et al., 2006. Acyl-phosphates initiate membrane phospholipid synthesis in Gram-positive pathogens. Mol. Cell, 23: 765-772. PMID: 16949372

Mazzoli, R., E. Pessione, M. Dufour, V. Laroute and M.G. Giuffrida et al., 2010. Glutamate-induced metabolic changes in Lactococcus lactis NCDO 2118 during GABA production: Combined transcriptomic and proteomic analysis. Amino. Acids, 39: 727-737. PMID: 20174841

Miajlovic, H. and S.G. Smith, 2014. Bacterial selfdefence: How Escherichia coli evades serum killing. FEMS Microbiol. Lett, 354: 1-9. PMID: 24617921

Moslehi-Jenabian, S., K. Gori and L. Jespersen, 2009. AI-2 signalling is induced by acidic shock in probiotic strains of Lactobacillus spp. Int. J. Food Microbi., 135: 295-302. PMID: 19748697

Plummer, P.J., 2012. LuxS and quorum-sensing in Campylobacter. Front Cell Infect. Microbiol., 2: 22. DOI: $10.3389 /$ fcimb.2012.00022

Rajan, R., J. Zhu, X. Hu, D. Pei and C.E. Bell, 2005. Crystal structure of S-ribosylhomocysteinase (LuxS) in complex with a catalytic 2-ketone intermediate. Biochemistry, 44: 3745-3753. DOI: $10.1021 / \mathrm{bi0} 047384$
Richard, H. and J.W. Foster, 2004. Escherichia coli glutamate- and arginine-dependent acid resistance systems increase internal $\mathrm{pH}$ and reverse transmembrane potential. J. Bacterio., 186: 60326041. DOI: 10.1128/JB.186.18.6032-6041.2004

Roy, H., 2009. Tuning the properties of the bacterial membrane with aminoacylated phosphatidylglycerol. Iubmb. Life, 61: 940-953. DOI: 10.1002/iub.240

Siragusa, S., M. De Angelis, R. Di Cagno, C.G. Rizzello and R. Coda et al., 2007. Synthesis of gamma-aminobutyric acid by lactic acid bacteria isolated from a variety of Italian cheeses. Applic. Environ. Microb, 73: 7283-7290. DOI: 10.1128/AEM.01064-07

Small, P.L. and S.R. Waterman, 1998. Acid stress, anaerobiosis and gadCB: Lessons from Lactococcus lactis and Escherichia coli. Trends Microbiol., 6: 214-216. DOI: 10.1016/S0966-842X(98)01285-2

Su, M.S., S. Schlicht and M.G. Gänzle, 2011. Contribution of glutamate decarboxylase in Lactobacillus reuteri to acid resistance and persistence in sourdough fermentation. Microb. Cell Fact, 10 1: S8. DOI: $10.1186 / 1475-2859-10-S 1-S 8$

Sun, Z., X. He, V.F. Brancaccio, J. Yuan and C.U. Riedel, 2014. Bifidobacteria exhibit LuxSdependent autoinducer 2 activity and biofilm formation. PLoS One, 9: e88260-e88260. DOI: 10.1371/journal.pone.0088260

Tramonti, A., M. De Canio, I. Delany, V. Scarlato and D. De Biase, 2006. Mechanisms of transcription activation exerted by $\mathrm{GadX}$ and GadW at the gadA and gadBC gene promoters of the glutamate-based acid resistance system in Escherichia coli. J. Bacteriol., 188: 8118-8127. DOI: 10.1128/JB.01044-06

Wouters, J.A., H.H. Kamphuis, J. Hugenholtz, O.P. Kuipers and T. Abee et al., 2000. Changes in glycolytic activity of Lactococcus lactis induced by low temperature. Applic. Environ. Microbiol., 66: 3686-3691. PMID: 10966377

Zamfir, M. and S. Grosu-Tudor, 2014. Stress response of some lactic acid bacteria isolated from Romanian artisan dairy products. World J. Microbiol. Biotechnol., 30: 375-384. DOI: $10.1007 / \mathrm{s} 11274-013-1454-6$

Zhang, Y. and M.W. Griffiths, 2003. Induced expression of the heat shock protein genes uspA and $g r p E$ during starvation at low temperatures and their influence on thermal resistance of Escherichia coli O157:H7. J. Food Prot., 66: 20452050. PMID: 14627281

Zininga, T. and A. Shonhai, 2014. Are heat shock proteins druggable candidates? Am. J. Biochem. Biotechnol., 10: 211-213. DOI: 10.3844/ajbbsp.2014.211.213 